\title{
Parameterization of travelling waves in plane Poiseuille flow
}

\author{
WARREN R. SMITH \\ School of Mathematics, University of Birmingham, Edgbaston, \\ Birmingham, B15 2TT, UK
}

AND

JAN G. WISSINK

School of Engineering and Design, Brunel University, Uxbridge, Middlesex, UB8 3PH, UK

November 14, 2014

\begin{abstract}
The first finite-dimensional parameterization of a subset of the phase space of the Navier-Stokes equations is presented. Travelling waves in two-dimensional plane Poiseuille flow are shown numerically to approximate maximum-entropy configurations. In a coordinate system moving with the phase velocity, the enclosed body of the flow exhibits a hyperbolic sinusoidal relationship between the vorticity and stream function. The phase velocity and two amplitude parameters describe the stable manifold on the slow viscous time scale. This original parameterization provides a valuable visualization of this subset of the phase space of the Navier-Stokes equations. These new results provide physical insight into an important intermediate stage in the instability process of plane Poiseuille flow. Parameterization, plane Poiseuille flow, maximum-entropy configurations
\end{abstract}

\section{Introduction}

The maximum-entropy configuration is very interesting as it describes a subset of the infinite-dimensional phase space by a finite number of slowly varying parameters. However, these configurations have not been reported for the physically important wall-bounded shear flows; although, Orszag and Patera (1983) observed that the vorticity has an unknown dependence on the stream function in these flows and Jones and Montgomery (1994) anticipated some of our conclusions. In this article, we present the surprising discovery that a manifold of travelling waves in plane Poiseuille flow exhibits the two-sign Joyce-Montgomery equation. This novel result not only bridges the gap between the research taking place on ideal Euler maximum-entropy configurations 
and wall-bounded shear flows, but opens alternative routes to visualizing, understanding and modelling such flows.

Three motivations for this study are now presented. First, we consider the physical significance of the maximum-entropy configuration. Almost all real channel flows in medical, engineering and geophysical applications are in three dimensions; nevertheless, the two-dimensional travelling waves studied in this article are at least an important intermediate stage in the initial-value problems of plane Poiseuille flow. Orszag and Patera (1983) described the instability process in three steps:

(i) primary (linear) instability of the basic shear flow;

(ii) nonlinear saturation of the primary instability and formation of a secondary flow;

(iii) secondary instability.

The two-dimensional travelling waves (or secondary flow) studied here act as saddles in phase space: the flow slowly approaches the steady state along the stable manifold (in step (ii)) before departing rapidly in the direction of the unstable manifold (in step (iii)). In this article, the two-dimensional flow (in step (ii)) is shown to be maximizing its entropy subject to the constraints of slowly varying total kinetic energy, total positive and negative vorticity. This new physical insight assists in our understanding of the persistence of the two-dimensional quasi-steady states in step (ii).

Secondly, asymptotic solutions of stable travelling waves are rare in wall-bounded shear flows. Any such solution is a valuable tool in checking the accuracy of the results of computational fluid dynamics. The vorticity-stream function relationship derived in this article is shown to be accurate to at least the order of the reciprocal of the Reynolds number. In practice, numerical simulations produce a greater accuracy due to the elimination of the secular viscous terms by modulation equations.

Thirdly, two-dimensional flows play a useful role as a laboratory for developing new mathematical methods and understanding physical mechanisms. Stuart (1960) considered two-dimensional plane Poiseuille flow when introducing weakly nonlinear theory into fluid mechanics. The parameterization described below is essential in identifying the slowly varying parameters in the fluid flow, the ultimate aim being to combine this result with the emerging subject of modulation equations for strongly nonlinear problems in fluid mechanics (see Smith, 2007, 2010).

It has been established for many years that the simulation of the two-dimensional high-Reynolds-number Navier-Stokes equations with periodic boundary conditions may relax to a quasi-steady state whose kinetic energy decays at a rate which is approximately inversely proportional to the Reynolds number (see Matthaeus et al., 1991a,b; Montgomery et al., 1992). The quasi-steady state was first computed for an initially turbulent run at a Reynolds number above 14000 which showed a slowly varying hyperbolic sinusoidal dependence between the vorticity and stream function. Joyce and Montgomery (1973) and Montgomery and Joyce (1974) first predicted this dependence in the context of the mean-field treatment of ideal line vortices and it has subsequently become known as the two-sign Joyce-Montgomery equation (see, for example, Chorin, 1994). An explanation of why an ideal Euler maximum-entropy configuration should describe the long-time Navier-Stokes simulations remains an open problem. 
An alternative formulation of maximum-entropy configuration has also been introduced (see Robert and Sommeria, 1991, 1992; Miller et al., 1992) and compared with a Navier-Stokes decay (see Yin et al., 2003). This formulation models vorticity as finite-area, mutually exclusive convected regions. Two-dimensional high-Reynoldsnumber Navier-Stokes simulations with periodic boundary conditions have been investigated, but originating from initial conditions which consisted of large areas of nearly flat vorticity patches plus random noise. Quasi-steady states were found which exhibited a vorticity-stream function relationship consistent with this alternative maximumentropy configuration.

For two-dimensional plane Poiseuille flow with constant streamwise pressure gradient, linear instability of the basic flow occurs at a Reynolds number of 5772. The Reynolds number is defined as $R=\rho U_{c} h / \mu$, where $h$ is the channel half-width, $\mu$ the dynamic viscosity, $\rho$ the density and $U_{c}$ is given by $U_{c}=G h^{2} / 2 \mu$ in which $-G$ is the constant pressure gradient. The existence of neutral finite-amplitude travelling waves has been demonstrated numerically for a Reynolds number greater than approximately 2900 and a finite band of wavenumbers (see Herbert, 1976). At subcritical Reynolds numbers, there are either zero or two finite-amplitude travelling waves on this neutral surface. If there are two, then the lower-energy branch is unstable while the higherenergy branch is stable to periodic disturbances. Each initial condition within the basin of attraction of the stable branch first evolves on the short convective time scale to a manifold of quasi-steady travelling waves and then approaches the travelling wave on the slow viscous time scale (see Orszag and Patera, 1981).

Gibson et al. (2008) reported a novel technique for visualizing low-dimensional projections of trajectories in the infinite-dimensional phase space of the Navier-Stokes equations. This projection method is much more widely applicable than the approach described in this article, but it is numerical and unsuitable for use in an asymptotic analysis.

The dimensionless initial-boundary-value problem is formulated in Section 2 and Section 3 summarizes the computational scheme which has been adopted. In Section 4 , the validity of the proposed parameterization of phase space is analyzed using the numerical evidence from the computed trajectory. The trajectory in the stable manifold of travelling waves is then visualized using the parameterization of phase space. In Section 5, the parameterization is shown to be uniformly valid for all quasi-steady travelling waves, but invalid for Reynolds numbers above the Hopf bifurcation. Finally, Section 6 gives a brief discussion of the results.

\section{Mathematical model}

The two-dimensional Navier-Stokes and continuity equations may be written

$$
\frac{\partial q}{\partial t}+(q \cdot \nabla) q+\nabla p=\frac{1}{R}\left[\nabla^{2} q+2 \hat{x}\right], \quad \nabla \cdot q=0,
$$

in which $R$ is the Reynolds number, $(x, y)^{T}$ are the spatial coordinates, $t$ is time, $q$ is the velocity vector $(u, v)^{T}, p$ is pressure and $\hat{x}$ is the unit vector along the $x$-axis. The 
boundary conditions are

$$
q(x, \pm 1, t)=0, \quad q(0, y, t)=q(2 \pi / \alpha, y, t),
$$

where $\alpha$ is a constant streamwise wavenumber. An initial condition is adopted within the basin of attraction of the quasi-steady travelling waves (see Orszag and Patera, 1981), with $R=4000$ and $\alpha=1.25$.

\section{Numerical method}

The two-dimensional incompressible Navier-Stokes equations are discretized on a uniform staggered mesh using a fourth-order accurate, kinetic energy conserving discretization of the convective terms (see Wissink, 2004) combined with a sixth-orderaccurate central discretization of the diffusive terms and a second-order accurate discretization of the Poisson equation for the pressure. The time integration is performed using the second-order-accurate Adams-Bashforth method using a non-dimensional time step of $\delta t=0.0002$. The results presented in this article are obtained on a $200 \times 200$ point mesh.

As an initial condition, disturbances are added to the Poiseuille flow profile

$$
q=\left(1-y^{2}\right)(1,0.1 \sin (\alpha x))+\left(0, v^{\prime}\right),
$$

where $v^{\prime}$ are random disturbances with $\operatorname{rms}\left(v^{\prime}\right)=0.001$. After 100 time units of simulation, the velocity field is re-initialized by significantly boosting the fluctuations $q=\left(1-y^{2}, 0\right)+12.5\left(q-\left(1-y^{2}, 0\right)\right)$ which forced the flow to become turbulent. After about 1000 time units and every subsequent 100 time units, sets of two instantaneous flow fields (one time unit apart) are recorded.

\section{Results}

We now describe the evidence that the vorticity and stream function obey, to a good approximation, the local relationship

$$
\omega=A \sinh (B[\psi-U y]),
$$

where $\omega$ is the vorticity, $\psi$ is the stream function, $U$ is the slowly varying phase velocity and $A$ and $B$ are slowly varying amplitude parameters. The phase velocity is evaluated by considering two snapshots of velocity separated by one time unit. The two parameters $A$ and $B$ are then determined using a least-squares approximation in the enclosed body of the flow $|\psi-U y|<0.15$ and $|y|<0.6$, where a single-valued function exists between the vorticity and the stream function.

The simulation runs until the vorticity-stream function relationship becomes singlevalued in the body of the flow; this is designated $t=0$ and the first recording of data takes place. Fig. 1 shows two scatter plots of the computed modified stream function $\psi-U y$ versus the computed vorticity $\omega$, taken pointwise over space at time $t=2500$. Fig. 1(a) corresponds to $|\psi-U y|<0.15$ and $|y|<0.6$ and Fig. 1(b) to $\psi-U y>0.15$ 
and $|y|<0.6$, it being clear that the relationship is not single-valued in Fig. 1(b). The solid line is the least-squares fit to (4.1) using the data from $|\psi-U y|<0.15$. Fig. 2 is the associated contour plot of the modified stream function which illustrates that the enclosed body of the flow $(|\psi-U y|<0.15$ and $|y|<0.6)$ is between the counterrotating eddies. The cross correlation is defined to be

$$
C[a, b]=\frac{\langle(a-\langle a\rangle)(b-\langle b\rangle)\rangle}{\left[\left\langle(a-\langle a\rangle)^{2}\right\rangle\left\langle(b-\langle b\rangle)^{2}\right\rangle\right]^{1 / 2}},
$$

where the angle brackets denote a spatial average over the body of the flow. Table 1 shows computed values of the cross correlations $C[\omega, \sinh (B[\psi-U y])]$ at different times; a cross correlation of one would indicate a perfect proportionality. The cross correlation shows that vorticity-stream function relationship is extremely accurate throughout the simulation.

Every one hundred time units, the least-squares fit is repeated and the results are recorded. The three parameters $A, B$ and $U$ define the vorticity-stream function relationship. Fig. 3 illustrates a trajectory in the stable manifold of travelling waves in this three-dimensional parameter space; the manifold spirals towards its steady state. When $t \geq 2500$, Fig. 4(a) shows that $B \approx 4.67$ and the approach to the steady state is projected onto a two-dimensional parameter space. The evolution of $A$ and $U$ for $t \geq 0$ is shown in Fig. 4(b) and 5. The parameterization is an extremely appealing visualization of the phase space of the Navier-Stokes equations.

The accuracy of the results are assessed by performing a grid refinement study comprising two further simulations employing $150 \times 150$ and $300 \times 300$ point meshes. The approximations to the travelling wave of the Navier-Stokes equations obtained on the three meshes are found to be in very good agreement: the coarsest mesh (not illustrated here) approximation converged to amplitudes of $A=0.860$ and $B=4.70$ and a phase velocity of $U=0.3344$, while the $200 \times 200$ and $300 \times 300$ point mesh approximations converged to $(A, B, U)=(0.863,4.67,0.3348)$ and $(A, B, U)=(0.865,4.65,0.3352)$, respectively (see Fig. 5).

Jones and Montgomery (1994) obtained a travelling wave at $R=4000$ and $\alpha=1.25$ which is consistent with the simulations in this article. They also reported a travelling wave at $R=15000$ and $\alpha=1.5$. In this second case, the body of the flow corresponds to $|\psi-U y|<0.05$ and the parameterization is given by $(A, B, U)=(0.453,14.6,0.2315)$.

The hyperbolic sinusoidal relationship is derived by assuming a statistical-mechanical behaviour for the system which only conserves the total kinetic energy,

$$
\frac{1}{2} \int_{\Omega} q \cdot q \mathrm{~d} x \mathrm{~d} y
$$

and the total positive and negative vorticity,

$$
\int_{\Omega} \omega^{ \pm} \mathrm{d} x \mathrm{~d} y
$$

In this article, $\Omega$ is the two-dimensional region defined by $|\psi-U y|<0.15$ and $|y|<$ 0.6 . We have $\omega=\omega^{+}-\omega^{-}$, where $\omega^{+}\left(\omega^{-}\right)$is the positive (negative) vorticity. These integrals are evaluated using one-point Gaussian quadrature for the $200 \times 200$ simulation and their values are shown in Fig. 6. On this trajectory, these three integrals are 
not conserved, but vary slowly on a time scale which is approximately proportional to the Reynolds number. The total positive and negative vorticity are initially not equal, but their difference diminishes. It would appear that a local maximum of entropy has been reached at each time on this trajectory corresponding to the constraints of the slowly varying total kinetic energy, the total positive and negative vorticity. The stable manifold may be considered to be a sequence of quasi-steady travelling waves.

\section{Asymptotic analysis}

\subsection{Introduction}

We have confirmed numerically that quasi-steady travelling waves exhibit a statisticalmechanical behaviour of an information-theoretic kind in a moving frame of reference. This behaviour is consistent with the leading-order problem on the short time scale being the Euler equations, where the model is singularly perturbed in $\varepsilon=1 / R \ll 1$. At larger Reynolds number, the travelling wave becomes unstable and undergoes a supercritical bifurcation into a limit cycle. At still larger Reynolds number, the solution bifurcates again into a two-frequency torus (see Jiménez, 1990). In fact, Jiménez (1990) adopted constant volume flux instead of the constant pressure gradient used in this article. It remains to be established whether or not the body of the flow for these cycles and tori exhibit maximum-entropy configurations. Parameterization would be even more valuable in understanding the phase space and visualizing the different attractors of the Navier-Stokes equations in these cases. In this context, it may appear reasonable to investigate at higher Reynolds number above the Hopf bifurcation; however, the following asymptotic analysis will demonstrate that this is incorrect.

\subsection{Multi-phased waves}

After each bifurcation, the solution acquires a new frequency and phase, therefore multi-phased waves must be studied. We consider the enclosed body of the flow in which the leading-order problem is inviscid. We adopt $N$ fast phases $\theta_{(j)}$ defined by $\partial \theta_{(j)} / \partial x=k_{(j)}$ and $\partial \theta_{(j)} / \partial t=-\sigma_{(j)}(\tilde{t})$ and a slow time-scale $\tilde{t}=\varepsilon t$, where $k_{(j)}$ is a constant streamwise wavenumber and $\sigma_{(j)}$ is a local frequency. We introduce expansions of the form

$$
\begin{aligned}
u & \sim u_{0}\left(\theta_{(1)}, \theta_{(2)}, \ldots, \theta_{(N)}, y, \tilde{t}\right)+\varepsilon u_{1}\left(\theta_{(1)}, \theta_{(2)}, \ldots, \theta_{(N)}, y, \tilde{t}\right) \\
v & \sim v_{0}\left(\theta_{(1)}, \theta_{(2)}, \ldots, \theta_{(N)}, y, \tilde{t}\right)+\varepsilon v_{1}\left(\theta_{(1)}, \theta_{(2)}, \ldots, \theta_{(N)}, y, \tilde{t}\right) \\
p & \sim p_{0}\left(\theta_{(1)}, \theta_{(2)}, \ldots, \theta_{(N)}, y, \tilde{t}\right)+\varepsilon p_{1}\left(\theta_{(1)}, \theta_{(2)}, \ldots, \theta_{(N)}, y, \tilde{t}\right), \\
\omega & \sim \omega_{0}\left(\theta_{(1)}, \theta_{(2)}, \ldots, \theta_{(N)}, y, \tilde{t}\right)+\varepsilon \omega_{1}\left(\theta_{(1)}, \theta_{(2)}, \ldots, \theta_{(N)}, y, \tilde{t}\right),
\end{aligned}
$$

as $\varepsilon \rightarrow 0$. At leading order, we obtain

$$
\bar{L} u_{0}+\sum_{j=1}^{N} k_{(j)} \frac{\partial p_{0}}{\partial \theta_{(j)}}=0
$$




$$
\begin{gathered}
\bar{L} v_{0}+\frac{\partial p_{0}}{\partial y}=0 \\
\sum_{j=1}^{N} k_{(j)} \frac{\partial u_{0}}{\partial \theta_{(j)}}+\frac{\partial v_{0}}{\partial y}=0,
\end{gathered}
$$

with the differential operator

$$
\bar{L}=\sum_{j=1}^{N}\left(k_{(j)} u_{0}-\sigma_{(j)}\right) \frac{\partial}{\partial \theta_{(j)}}+v_{0} \frac{\partial}{\partial y} .
$$

We take the curl of (5.1a)-(5.1b) to obtain $\bar{L} \omega_{0}=0$ where the leading-order vorticity $\omega_{0}$ is given by

$$
\omega_{0}=\sum_{j=1}^{N} k_{(j)} \frac{\partial v_{0}}{\partial \theta_{(j)}}-\frac{\partial u_{0}}{\partial y} .
$$

A stream function $\bar{\psi}$ is defined by the equations

$$
u_{0}=\frac{\partial \bar{\psi}}{\partial y}, \quad v_{0}=-\sum_{j=1}^{N} k_{(j)} \frac{\partial \bar{\psi}}{\partial \theta_{(j)}},
$$

so that (5.1c) is satisfied and the vorticity equation may be rewritten as

$$
\sum_{j=1}^{N} k_{(j)} \frac{\partial}{\partial y}\left(\bar{\psi}-U_{(j)} y\right) \frac{\partial \omega_{0}}{\partial \theta_{(j)}}-\sum_{j=1}^{N} k_{(j)} \frac{\partial}{\partial \theta_{(j)}}\left(\bar{\psi}-U_{(j)} y\right) \frac{\partial \omega_{0}}{\partial y}=0,
$$

where $U_{(j)}=\sigma_{(j)} / k_{(j)}$.

In the case $N=1$, we have quasi-steady travelling waves. The first integral of (5.2) is a functional of the form

$$
\omega_{0}=V\left(\bar{\psi}-U_{(1)} y ; A(\tilde{t}), B(\tilde{t}), \ldots\right)
$$

where $A(\tilde{t}), B(\tilde{t}), \ldots$ are slowly varying parameters determined by modulation equations at next order. This functional was anticipated by Orszag and Patera (1983). In a coordinate system moving with the phase velocity and on the short time scale, (5.3) is independent of Reynolds number and aspect ratio. Therefore, if a functional holds for one Reynolds number and aspect ratio, then it holds for all combinations of Reynolds number and aspect ratio below the Hopf bifurcation. Hence, the parameterization (4.1) is uniformly valid for all quasi-steady travelling waves.

In the case $N=2$, we have limit cycles. A first integral of the form (5.3) only exists if the problem is degenerate. Thus, the parameterization (4.1) is invalid for Reynolds numbers above the Hopf bifurcation. Similar comments apply for $N>2$.

\section{Conclusions}

The parameters for quasi-steady travelling waves in plane Poiseuille flow arise from three sources, where $R \gg 1$ and the viscous terms are negligible at leading order in 
an enclosed body of the flow. The first source corresponds to the local frequency (or, equivalently, the phase velocity). The first integral of the Euler equations given by (4.1) requires two further parameters. The stream function is then determined by solving the sinh-Poisson equation, which would typically require an infinite number of additional parameters. We do not seek an exact representation of the stream function, because this solution of the Navier-Stokes equations is also a maximum-entropy configuration. The three parameters $A, B$ and $U$ are sufficient to characterize the nature of the solution because (4.1) defines the maximum-entropy configuration.

There are four principal differences between the two-dimensional decay with periodic boundary conditions and the one reported here: (i) the phase velocity is an additional parameter; (ii) we approach a travelling wave with non-zero kinetic energy; (iii) there is no possibility of an alternative maximum-entropy configuration (see Robert and Sommeria, 1991, 1992; Miller et al., 1992; Yin et al., 2003) as the hyperbolic sinusoidal relationship is a property of our travelling wave; and (iv) the total kinetic energy, positive and negative vorticity are all slowly varying in contrast to the spatially periodic domain where the vorticities were both exactly conserved (see Montgomery et al., 1992).

The stable steady state described in this article corresponds to a generalized saddle for the three-dimensional problem with periodic boundary conditions in the third dimension (see Orszag and Patera, 1983). Our two-dimensional results have established that this saddle and its two-dimensional manifold are parameterized by the two-sign Joyce-Montgomery equation. The intriguing possibility arises that the unstable steady states in other wall-bounded shear flows (for which a single vorticity and stream function may be defined) may also possess maximum-entropy configurations. The examination of the time evolution of kinetic energy, positive and negative vorticity in the inviscid body of these flows may be employed to flag up the occurrences of these configurations.

In conclusion, ideal Euler maximum-entropy configurations have been numerically shown to exist in plane Poiseuille flow. It proved necessary to adopt a coordinate system moving with the slowly varying phase velocity in order to reveal the hyperbolic sinusoidal relationship between the vorticity and stream function. The resulting slowly varying parameters and phase velocity described the stable manifold on the viscous time scale. This finite-dimensional parameterization has not been previously discovered in the description of travelling waves in wall-bounded shear flows.

\section{References}

Chorin, A. J. (1994). Vorticity and Turbulence. Springer-Verlag, New York.

Gibson, J. F., Halcrow, J., and Cvitanović, P. (2008). Visualizing the geometry of state-space in plane Couette flow. J. Fluid Mech., 611, 107-130.

Herbert, T. (1976). Periodic secondary motions in a plane channel. In A. I. Van de Vooren and P. J. Zandbergen, editors, Proc. 5th Int. Conf. on Numerical Methods in Fluid Dynamics, pages 235-240. Springer. Lecture Notes in Physics, vol 59. 
Jiménez, J. (1990). Transition to turbulence in two-dimensional Poiseuille flow. $J$. Fluid Mech., 218, 265-297.

Jones, W. B. and Montgomery, D. (1994). Finite amplitude steady states of high Reynolds number 2-D channel flow. Physica D, 73, 227-243.

Joyce, G. R. and Montgomery, D. (1973). Negative temperature states for a twodimensional guiding-centre plasma. J. Plasma Phys., 10, 107-121.

Matthaeus, W. H., Stribling, W. T., Martinez, D., Oughton, S., and Montgomery, D. (1991a). Decaying, two-dimensional, Navier-Stokes turbulence at very long times. Physica D, 51, 531-538.

Matthaeus, W. H., Stribling, W. T., Martinez, D., Oughton, S., and Montgomery, D. (1991b). Selective decay and coherent vortices in two-dimensional incompressible turbulence. Phys. Rev. Lett., 66, 2731-2734.

Miller, J., Weichman, P. C., and Cross, M. C. (1992). Statistical mechanics, Euler's equation and Jupiter's red spot. Phys. Rev. A, 45, 2328-2361.

Montgomery, D. and Joyce, G. R. (1974). Statistical mechanics of negative temperature states. Phys. Fluids, 17, 1139-1145.

Montgomery, D., Matthaeus, W. H., Stribling, W. T., Martinez, D., and Oughton, S. (1992). Relaxation in two dimensions and the "sinh-Poisson" equation. Phys. Fluids A, 4, 3-6.

Orszag, S. A. and Patera, A. T. (1981). Subcritical transition to turbulence in planar shear flows. In R. E. Meyer, editor, Transition and Turbulence, pages 127-146. Academic Press, New York.

Orszag, S. A. and Patera, A. T. (1983). Secondary instability of wall-bounded shear flows. J. Fluid Mech., 128, 347-385.

Robert, R. and Sommeria, J. (1991). Statistical equilibrium states for two-dimensional flows. J. Fluid Mech., 229, 291-310.

Robert, R. and Sommeria, J. (1992). Relaxation towards a statistical equilibrium state in two-dimensional perfect fluid mechanics. Phys. Rev. Lett., 69, 2776-2779.

Smith, W. R. (2007). Modulation equations and Reynolds averaging for finiteamplitude nonlinear waves in an incompressible fluid. IMA J. Appl. Math., 72, 923-945.

Smith, W. R. (2010). Modulation equations for strongly nonlinear oscillations of an incompressible viscous drop. J. Fluid Mech., 654, 141-159.

Stuart, J. T. (1960). On the nonlinear mechanics of wave disturbances in stable and unstable parallel flows. Part 1. The basic behaviour in plane Poiseuille flow. J. Fluid Mech., 9, 353-370. 
Wissink, J. G. (2004). On unconditional conservation of kinetic energy by finitedifference discretizations of the linear and non-linear convection equation. Computers and Fluids, 33, 315-343.

Yin, Z., Montgomery, D. C., and Clercx, H. J. H. (2003). Alternative statisticalmechanical descriptions of decaying two-dimensional turbulence in terms of "patches" and "points". Phys. Fluids, 15, 1937-1953. 
Selected times Spatially averaged cross correlation

$\begin{array}{cc}t & C[\omega, \sinh (B[\psi-U y])] \\ 0 & 0.999991 \\ 100 & 0.999995 \\ 200 & 0.999997 \\ 300 & 0.999998 \\ 400 & 0.999999 \\ 1000 & 1.000000 \\ 2500 & 0.999999 \\ 4000 & 0.999999 \\ 6200 & 0.999999\end{array}$

Table 1: The time evolution of the spatially averaged cross correlation (4.2).

(a)
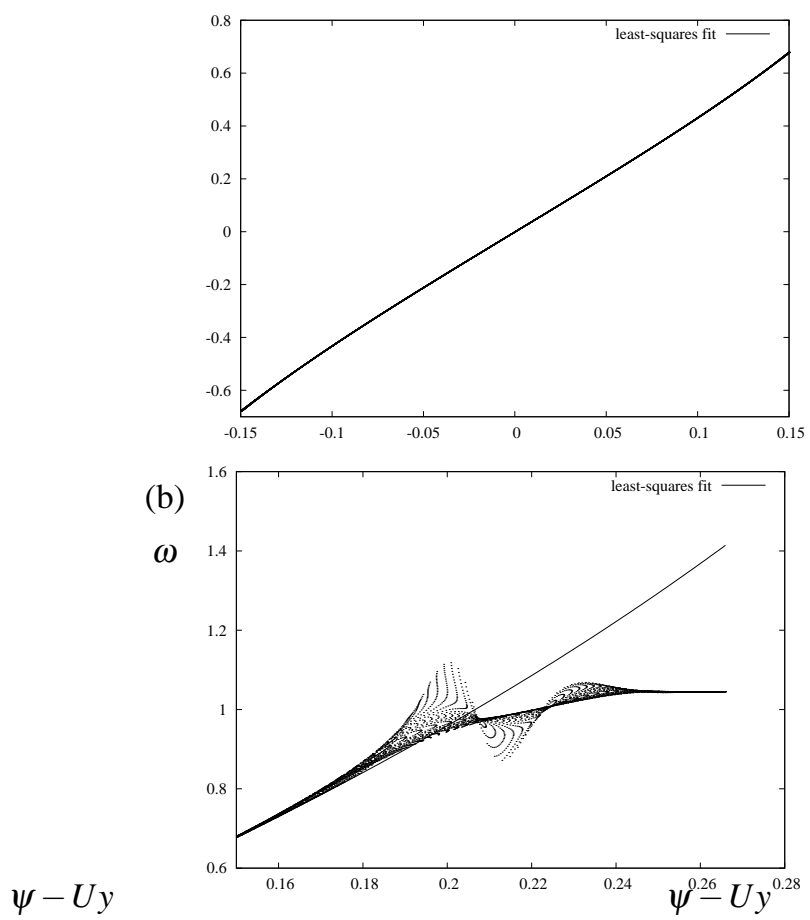

Figure 1: Scatter plots of the modified stream function $\psi-U y$ versus the vorticity $\omega$ at time $t=2500$ for $(a)|\psi-U y|<0.15$ and $|y|<0.6$ and $(b) \psi-U y>0.15$ and $|y|<0.6$. The curve drawn through the plotted points is $\omega=A \sinh (B[\psi-U y])$. 


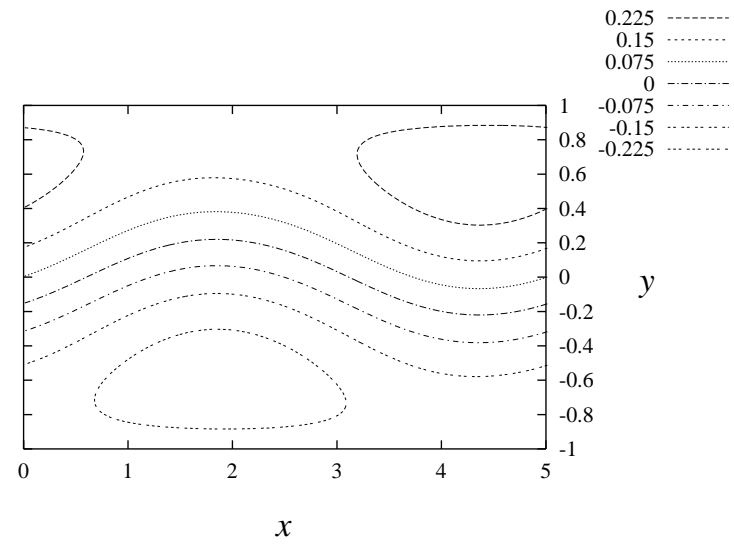

Figure 2: Three-dimensional contour plot of the modified stream function $\psi-U y$ as a function of $x$ and $y$ at $t=2500$. The enclosed body of the flow $(|\psi-U y|<0.15$ and $|y|<0.6)$ is between the counter-rotating eddies.

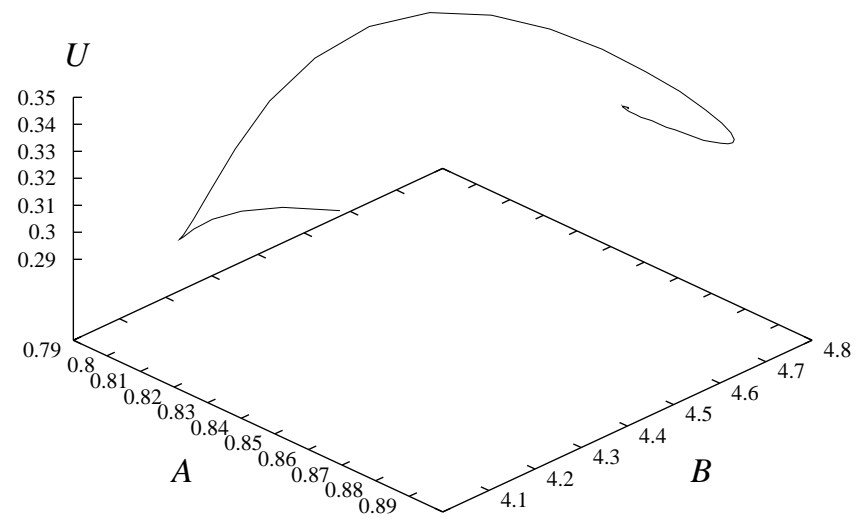

Figure 3: A trajectory in the stable manifold of travelling waves visualized using the parameterization of phase space. The steady state corresponds to a generalized focus. 


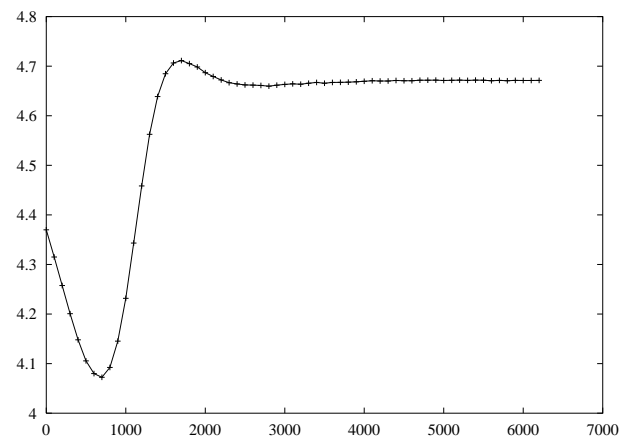

(a)

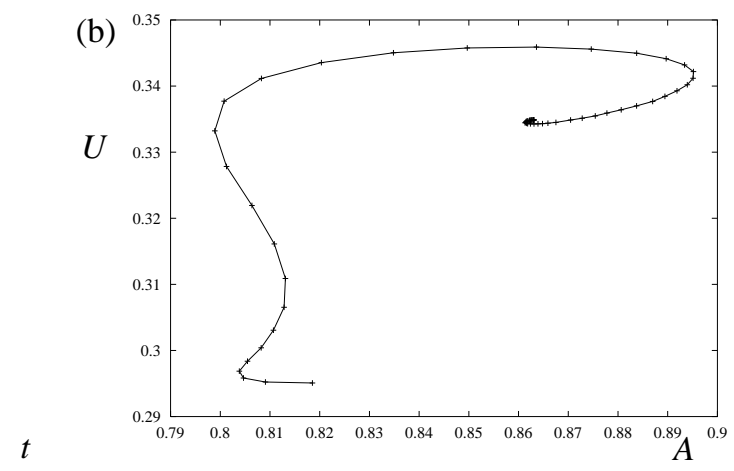

Figure 4: $(a)$ The slow variation of $B$ with time $t$ and $(b)$ the projection in the $(A, U)$ plane.

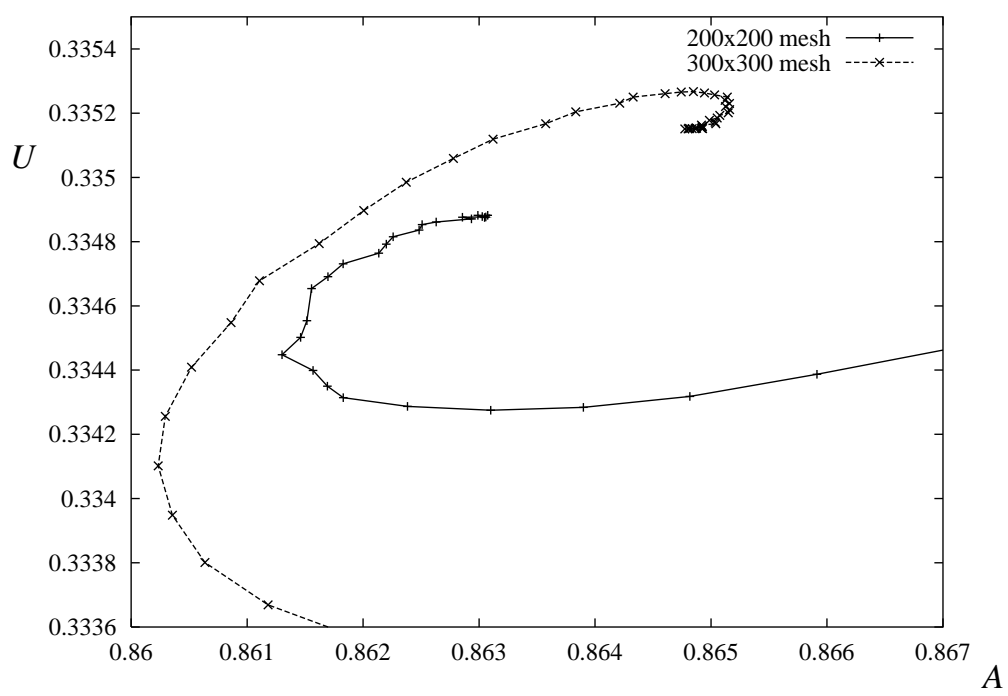

Figure 5: An enlargement of the projection in the $(A, U)$-plane near the steady state. The two simulations have different initial conditions and meshes. 


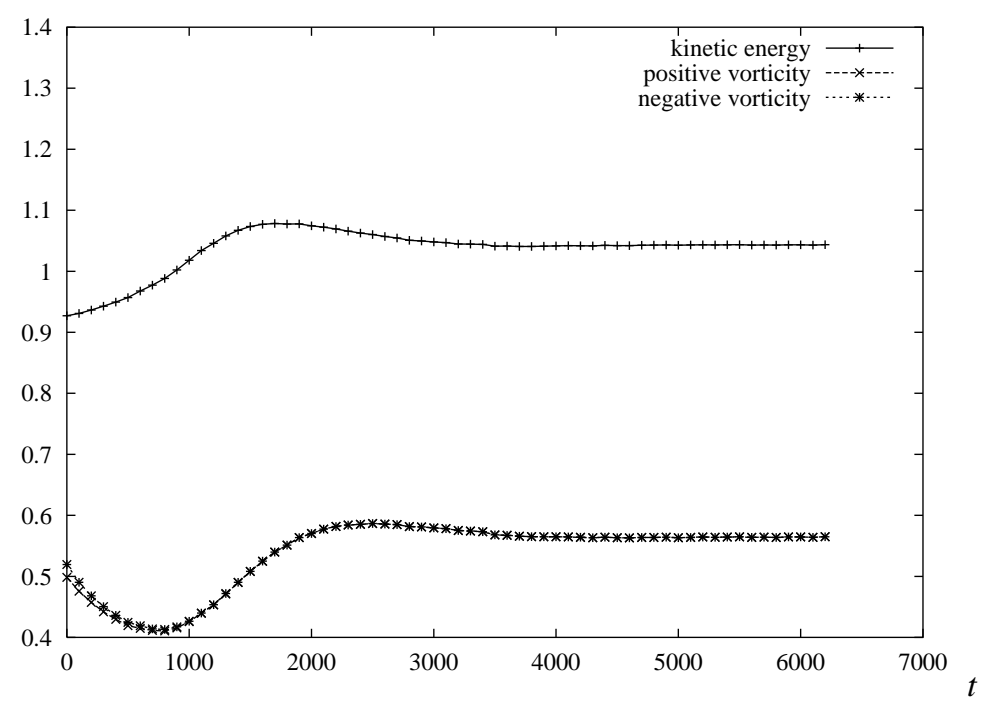

Figure 6: The slow variation of total kinetic energy $\int_{\Omega} q \cdot q \mathrm{~d} x \mathrm{~d} y / 2$, total positive and negative vorticity $\int_{\Omega} \omega^{ \pm} \mathrm{d} x \mathrm{~d} y$ with time $t$ for the $200 \times 200$ mesh. 